\title{
Contribuições da simulação clínica versus prática convencional em laboratório de enfermagem na primeira experiência clínica
}

\author{
Contributions of clinical simulation versus conventional practice in a nursing laboratory in the first clinical \\ experience
}

\section{Contribuciones de la simulación clínica versus la práctica convencional en laboratorio de enfermería en} la primera experiencia clínica

\author{
Radamés Boostel ${ }^{1}$ (10 \\ Carina Bortolato-Major ${ }^{2}$ (1) \\ Nilton Orlando da Silva ${ }^{1}$ (1) \\ Jéssica de Oliveira Veloso Vilarinho ${ }^{1}$ (B) \\ Amanda Carolina de Oliveira Bialetzki \\ Fontoura ${ }^{1}$ (1) \\ Jorge Vinícius Cestari Felix ${ }^{1}$ [C]
}

1. Universidade Federal do Paraná, Programa de Pós-Graduação em Enfermagem. Curitiba, PR, Brasil.

2. Universidade Estadual do Norte do Paraná, Setor de Enfermagem. Bandeirantes, PR, Brasil.

Autor correspondente:

Radamés Boostel.

E-mail: radames.boostel@gmail.com.

Recebido em 04/08/2020.

Aprovado em 22/12/2020

DOI:https://doi.org/10.1590/2177-9465-EAN-2020-0301

\begin{abstract}
Resumo
Objetivo: Comparar a percepção de estudantes de enfermagem e as contribuições do ensino com simulação clínica ou aula prática convencional em laboratório de habilidades, na primeira experiência clínica hospitalar. Método: Pesquisa descritiva, qualitativa, envolvendo estudantes de graduação em enfermagem de uma universidade pública do Brasil, submetidos à simulação clínica de alta fidelidade ou aula prática convencional, ocorrida entre 2015 e 2016. Para avaliação dos dados, utilizaram-se a nuvem de palavras e a análise de similitude do software IRAMUTEQ ${ }^{\circ}$. Resultados: Participaram 54 estudantes, sendo 27 em cada grupo. As palavras mais evocadas pelo grupo simulação foram: "paciente real, não, mais e simulador", relacionadas à capacidade de reflexão quanto ao seu conhecimento e preparo. As mais evocadas no grupo prática convencional foram: "mais, não, muito e sentir", relacionadas com a percepção de que a aula convencional ajuda na aquisição de habilidades, contudo, é necessária maior frequência. Conclusão: As duas estratégias contribuíram positivamente para a primeira experiência clínica hospitalar. Porém, a simulação proporcionou uma visão crítico-reflexiva sobre as competências, deficiências e maior autoconfiança em relação à prática convencional. Este estudo fortalece as evidências dos benefícios proporcionados pelo ensino baseado em simulação, e a importância de instituições de ensino fazerem uso adequado dessa estratégia.
\end{abstract}

Palavras-chave: Simulação; Treinamento por Simulação; Estudantes de Enfermagem; Aprendizagem; Ensino.

\begin{abstract}
Objective: to compare the perception of nursing students and the contributions of teaching with clinical simulation or conventiona practical classroom skills in the first clinical hospital experience. Method: a descriptive, qualitative research involving undergraduate nursing students from a public university in Brazil, submitted to a high fidelity clinical simulation or conventional practical class which took place between 2015 and 2016. For data evaluation, a word cloud and similarity analysis of the IRAMUTEQ $尺$ software were used. Results: Altogether 54 students participated, 27 of them in each group. The words most evoked by the simulation group were: "real patient, no, more and simulator", related to the capacity of reflection regarding their knowledge and preparation. The most evoked by the conventional practice group were: "more, no, much and feel", related with the perception that the conventiona class helps in the acquisition of skills, however, more frequency is needed. Conclusion: Both strategies contributed positively to the first clinical hospital experience. However, the simulation provided a critical-reflexive view of skills, deficiencies and greater self-confidence in relation to conventional practice. This study strengthens the evidence of the benefits provided by simulationbased teaching, and the importance of teaching institutions making appropriate use of this strategy.
\end{abstract}

Keywords: Simulation Technique; Simulation Training; Students, Nursing; Learning; Teaching

\section{Resumen}

Objetivo: Comparar la percepción de los estudiantes de enfermería y las contribuciones docentes con simulación clínica o clase práctica convencional en un laboratorio de habilidades, en la primera experiencia clínica hospitalaria. Métodos: Investigación descriptiva y cualitativa con estudiantes universitarios de enfermería en una universidad pública de Brasil, sometidos a simulación clínica de alta fidelidad o clase práctica convencional, entre 2015 y 2016 . Para el análisis de los datos se utilizo, una nube de palabras y el análisis de la similitud por el software IRAMUTEQ. Resultados: Participaron 54 estudiantes, 27 en cada grupo. Las palabras mas evocadas por el grupo de simulación fueron: "paciente real, no, más y simulador" relacionadas con la capacidad de reflexionar sobre su conocimiento y preparación. Los más mencionados en el grupo práctica convencional fueron: "más, no, mucho y sentir", relacionadas con la percepción de que la clase convencional ayuda en la adquisición de habilidades, sin embargo se necesita más frecuencia. Conclusion: Ambas estrategias contribuyeron positivamente para la primera experiencia clínica hospitalaria. Sin embargo, la simulación proporcionó una visión crítica reflexiva de las habilidades deficiencias y una mayor autoconfianza en comparación con la práctica convencional. Este estudio fortalece la evidencia de los beneficios proporcionados por la enseñanza basada en simulación y la importancia de que las instituciones educativas hagan uso adecuado de esta estrategia.

Palabras clave: Simulacion; Entrenamiento Simulado; Estudiantes de Enfermería; Aprendizaje; Enseñanza. 


\section{INTRODUÇÃO}

A prática clínica é considerada parte essencial e integrante dos cursos de graduação em enfermagem, pois é nesse momento que os estudantes aplicam e desenvolvem o conhecimento, as habilidades e as diferentes competências adquiridas em sala de aula ou laboratório. Também no início dessa fase observa-se maior interesse ou rejeição pela profissão em decorrência dos sentimentos, expectativas e desafios enfrentados..$^{1,2}$

A primeira experiência clínica geralmente é acompanhada de grandes expectativas e incertezas, as quais podem provocar sentimentos positivos, ou dificultar o processo de aprendizagem e deixar o estudante suscetível a sensações de incapacidade, angústia, tristeza, constrangimento, insegurança, raiva, medo, ansiedade. ${ }^{2}$ Tal fato demonstra a necessidade de os professores adotarem metodologias que favoreçam o aprendizado, e medidas que minimizem esses sentimentos durante a formação. ${ }^{3}$

Nesse contexto, a integração de novas tecnologias e metodologias educacionais na prática docente em enfermagem tem se mostrado relevante na evolução do processo de ensinoaprendizagem. Entre elas, observa-se o aumento do uso da simulação clínica (SC) como estratégia de ensino, a qual se revelou eficaz, pois favorece o desenvolvimento de habilidades e competências, auxilia na formação do pensamento crítico, raciocínio e julgamento clínicos, melhora a autoconfiança e satisfação dos estudantes. Além disso, contribui para a segurança do paciente e permite ao facilitador aplicar uma variedade de casos clínicos em um cenário de prática semelhante ao real, antes do contato com o paciente. - $^{-8}$

A satisfação dos estudantes é primordial para a manutenção de um bom ambiente de aprendizagem, e imprescindível ao desenvolvimento de competências por meio da prática simulada., ${ }^{6,-11}$ A melhora cognitiva relacionada à satisfação é um componente essencial da motivação acadêmica e indicador importante na percepção da aprendizagem. ${ }^{12}$

$\mathrm{Na}$ última década, estudos nacionais e internacionais demonstraram que o treinamento por simulação melhora o desenvolvimento de competência clínica, provoca a curiosidade epistemológica, diminui a insegurança e a ocorrência de eventos adversos causados durante a formação clínica. ${ }^{13,14}$

Apesar de os estudos internacionais demonstrarem fortes evidências sobre os benefícios do uso da simulação clínica, são necessários estudos adicionais para a compreensão dos efeitos dessa estratégia de ensino na realidade brasileira. $\mathrm{O}$ objetivo deste estudo foi conhecer a percepção dos estudantes de enfermagem e as contribuições do uso da simulação clínica de alta fidelidade, comparando-a com a aula prática convencional em laboratório de habilidades e após a primeira experiência clínica em ambiente hospitalar.

\section{MÉTODO}

Trata-se de uma pesquisa descritiva, de abordagem qualitativa, que comparou a percepção de estudantes de enfermagem submetidos à simulação clínica de alta fidelidade com a de discentes que participaram de aula prática convencional em laboratório de habilidades.

A amostra foi composta por estudantes do quarto período de graduação em enfermagem de uma universidade pública do sul do Brasil, entre os meses de agosto de 2015 e setembro de 2016. O pesquisador realizou o convite para participação na pesquisa pessoalmente, em sala de aula, no início do semestre letivo, momento em que explicou os seus objetivos e esclareceu as dúvidas existentes.

Considerou-se como critério de inclusão estar matriculado na disciplina Fundamentos para o Cuidar em Enfermagem. Os critérios de exclusão foram: formação em outro curso de graduação na área da saúde, com competência para exame físico, ou formação de auxiliar/técnico em enfermagem; alunos com histórico de trancamento ou reprovação na referida disciplina.

A coleta de dados ocorreu em dois momentos: após as aulas práticas em laboratório de habilidades ou laboratório de simulação clínica de alta fidelidade; e em ambiente hospitalar depois da primeira experiência clínica com pacientes. O conteúdo teóricoprático envolvia a anamnese e o exame físico cardiotorácico. Anterior à coleta de dados, os estudantes foram novamente orientados sobre os objetivos da pesquisa, e participaram de uma aula teórica referente à temática aplicada pelo professor da disciplina, em conjunto com o pesquisador principal.

Posteriormente à assinatura do Termo de Consentimento Livre e Esclarecido (TCLE), o pesquisador principal criou uma listagem aleatória com os nomes dos estudantes. Na sequência, utilizou-se a função aleatório-entre do programa Microsoft Exce/® para realizar a aleatorização. Os participantes foram incluídos um a um, e o programa os alocou no Grupo de Aula Prática Convencional em Laboratório de Habilidades (GPC) ou no Grupo Simulação Clínica (GSC).

GPC - os participantes realizavam a anamnese e o exame físico em outro estudante escolhido por eles, de acordo com o grau de afinidade. A aula de habilidades seguiu o roteiro estabelecido no plano de aula pelo professor responsável pela disciplina. As habilidades envolviam avaliação do pulso, circunferência do braço, aferição da pressão arterial em repouso e em atividade, ausculta cardíaca e avaliação do tórax.

GSC - um cenário de simulação clínica de alta fidelidade foi elaborado de acordo com o modelo de simulação da National League of Nursing/Jeffries. ${ }^{15}$ Os participantes deveriam proceder ao atendimento de paciente com quadro de dor torácica que estava internado em um leito de enfermaria. $O$ objetivo principal era a realização da anamnese e exame físico com foco cardiorrespiratório. A simulação envolveu cinco minutos para o briefing. Os participantes receberam orientações quanto às questões éticas e de confidencialidade da SC, e que a SC não era critério de avaliação da disciplina; conheceram o ambiente, as funcionalidades do simulador e receberam a história e o prontuário com dados do paciente. $\mathrm{O}$ cenário teve duração de quinze minutos, sendo utilizado o simulador de alta fidelidade METIman $®$. No início do cenário, o paciente encontrava-se deitado no leito, acordado e com os sinais vitais dentro da 
normalidade. Após os estudantes realizarem a anamnese e a avaliação da ausculta, frequência e ritmo cardíaco, e a pressão arterial, o paciente queixou-se de mal-estar e solicitou nova avaliação. Nesse momento, a frequência cardíaca e a pressão arterial foram alteradas. O debriefing estruturado foi realizado pelo pesquisador principal e teve duração de 30 minutos.

Antes da simulação, os acadêmicos desenvolveram práticas em laboratório de habilidades, conforme o praticado pelo GPC. As diferentes estratégias de ensino com os grupos foram executadas em dias consecutivos, a fim de evitar possíveis interferências de comunicação. A primeira experiência clínica ocorreu no mesmo dia para ambos os grupos, em um hospital-escola público, nos setores de clínica médica masculina e feminina, clínica cirúrgica, clínica neurológica, e no setor de internamento de uma Unidade de Pronto Atendimento (UPA).

Os dois grupos responderam, por escrito, a três questões sobre a estratégia de ensino utilizada e sua contribuição no primeiro contato com o paciente: "Como foi a experiência desta aula para você?"; "Você se sente preparado(a) para a sua primeira experiência clínica com o paciente real?"; "Você considera que a aula em laboratório ou simulação facilitou o seu primeiro contato com o paciente? Por quê?".

As questões foram elaboradas pelo pesquisador principal junto com o professor da disciplina Fundamentos para o Cuidar em Enfermagem. Visando garantir a privacidade dos participantes, optou-se pela utilização de código alfanumérico, em que " $A$ " correspondente a aluno.

Após o fim da coleta de dados, foi ofertada ao GPC uma aula de simulação igual à do GSC, no intuito de possibilitar experiência semelhante para todos e evitar eventuais prejuízos.

Para a análise dos dados, utilizou-se o software IRAMUTEQ® (Interface de $R$ pour les Analyses Multidimensionnelles de Textes et de Questionnaires) 0.7 alpha 2, desenvolvido por Pierre Ratinaud. ${ }^{16} \mathrm{O}$ corpus de texto foi construído a partir do agrupamento das respostas de cada estudante para as três perguntas, os quais foram codificados por letras e números. Realizaram-se análises por "nuvem de palavras" e "análise de similitude"16. A nuvem organiza e agrupa graficamente as palavras segundo a frequência com que aparecem no corpus. A análise de similitude apresenta as palavras conforme suas conexões textuais e permite identificar as coocorrências.

O estudo foi aprovado pelo Comitê de Ética em Pesquisa de uma universidade federal do sul do país, no mês de março

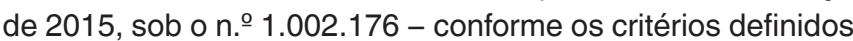

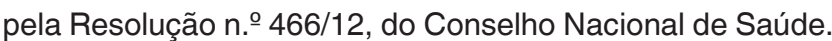

\section{RESULTADOS}

Aceitaram participar da pesquisa 54 estudantes de um total de 63 elegíveis, sendo alocados, inicialmente, 27 em cada grupo. Após o início da coleta de dados, dois participantes do GPC foram excluídos: um por desistência do curso antes da primeira experiência clínica e outro por não responder a uma das perguntas. A média de idade do GSC foi de 20,32 $\pm 1,79$ anos e do GPC, $21,11 \pm 2,47$ anos. O sexo predominante para ambos os grupos foi o feminino (85,19\% - GSC; $92,00 \%$ - GPC). Quanto às experiências prévias com simulação clínica, $100 \%$ dos participantes relataram nunca terem participado de simulação.

Na Tabela 1, são apresentados os resultados numéricos da análise dos corpora textuais de ambos os grupos.

Ao analisarmos as nuvens de palavras obtidas pelas respostas dos participantes (Figura 1), verificamos que as mais evocadas pelo GSC foram: "paciente real, não, mais e simulador" com 55, 48, 45 e 42 ocorrências, respectivamente. Essas palavras correspondem ao momento de reflexão e aprendizado que os estudantes tiveram ao experimentar a simulação.

No simulador, pudemos treinar a apresentação pessoal que com colegas não funciona direito; o simulador também me fez preparar para o improviso, responder dúvidas do paciente real e melhorar essa interação. (A6)

O simulador de alta fidelidade proporcionou um momento de reflexão a respeito das minhas atitudes perante $o$ paciente real e a equipe, além da minha forma de agir perante as minhas dificuldades. (A48)

Não, eu precisaria treinar mais vezes para me sentir mais preparada para interagir com o paciente real. (A27)

No GPC, as ocorrências mais observadas foram: "mais, não, muito, e sentir", com 40, 31, 25 e 24 vezes. Apesar de não terem experimentado a simulação, o GPC considerou a aula prática convencional um momento de preparação para a primeira experiência clínica, que também gerou confiança e segurança, além da percepção da necessidade de melhorar o conhecimento:

Sinto que as coisas não são difíceis, mas que é preciso prática. (A8)

A gente aprendendo e praticando com os colegas de turma torna mais fácil o contato com o paciente real, eu me senti segura por já ter tido a experiência de examinar alguém mesmo que não seja um paciente. (A13)

Tabela 1. Resultados das análises dos corpora textuais.

\begin{tabular}{lcc}
\hline & $\begin{array}{c}\text { Grupo Simulação } \\
\text { Clínica }\end{array}$ & $\begin{array}{c}\text { Grupo Prática } \\
\text { Convencional }\end{array}$ \\
\hline Número de textos & 27 & 25 \\
\hline $\begin{array}{l}\text { Número de } \\
\text { segmentos de texto }\end{array}$ & 70 & 54 \\
\hline Ocorrências & 2578 & 1818 \\
\hline Número de formas & 649 & 509 \\
\hline Número de hápax & 361 & 295 \\
& $\begin{array}{c}55,62 \% \text { das } \\
\text { formas }\end{array}$ & $\begin{array}{c}57,96 \% \text { das } \\
\text { formas }\end{array}$ \\
& $\begin{array}{l}14,00 \% \text { das } \\
\text { ocorrências }\end{array}$ & $\begin{array}{l}16,23 \% \text { das } \\
\text { ocorrências }\end{array}$ \\
\hline
\end{tabular}

Fonte: Os autores (2017). 


\section{Grupo Simulação Clínica}

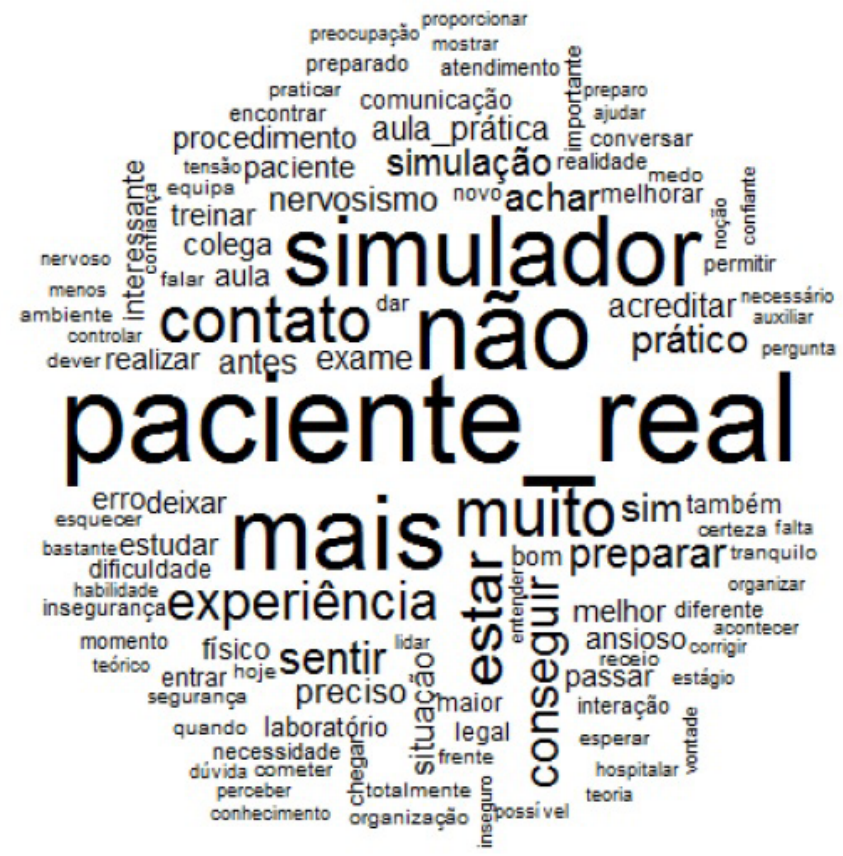

\section{Grupo Prática Convencional}

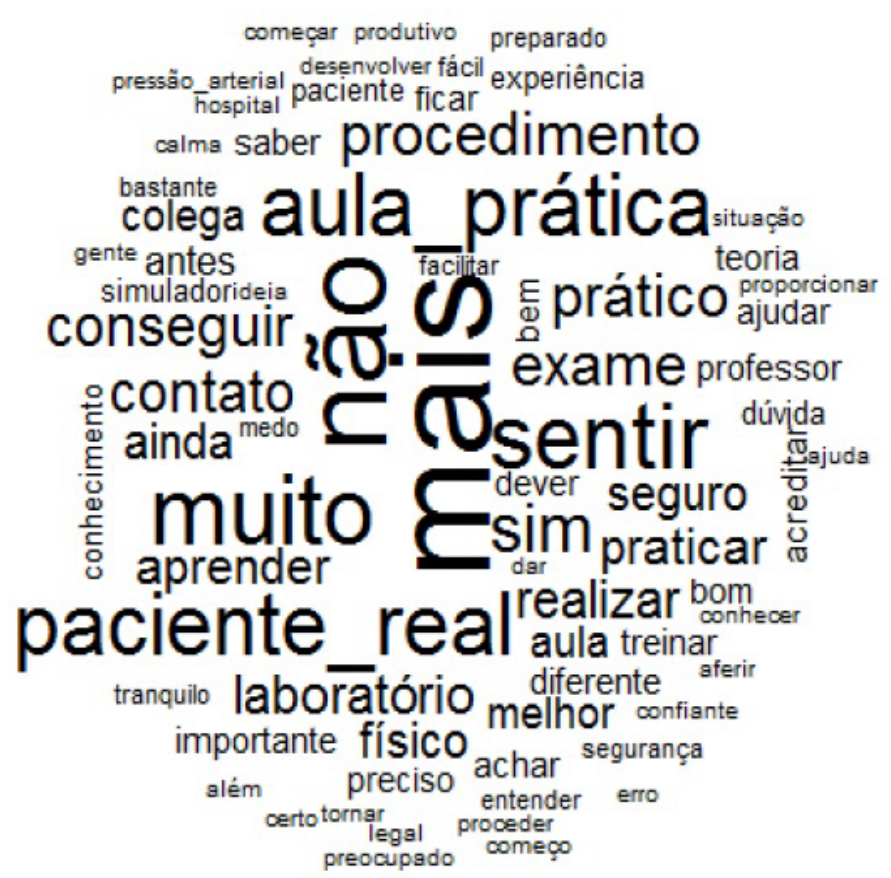

Figura 1. Comparação da nuvem de palavras dos corpora dos grupos. Curitiba, PR, Brasil, 2017.

Fonte: Os autores (2017).

... sinto que preciso de mais aula prática e teóricas antes de atender um paciente real. (A17)

A árvore de similitude do GSC (Figura 2) apresentou quatro itens lexicais principais: "paciente real, não, mais, simulador", tendo o termo "paciente real" apresentado maior número de conectividades. Palavras como "experiência, preparo, treinar, conseguir, permitir, organizar e contato" possuem proximidade com a expressão "paciente real". A palavra "mais" possui conexão com "paciente real e simulador" e ligações fortes e próximas com os termos "preciso, realidade, confiante, aula, acreditar, situação e nervosismo".

O item lexical "simulador" está na periferia do termo "paciente real"; entretanto, possui fortes afinidades com as palavras "interessante, aula prática, colega, erro, estar, nervoso, legal e dificuldade". No GSC, o item lexical "não" possui forte conectividade com "paciente real" e proximidade com as palavras "preparado, interação, muito, dever, bom”, entre outras.

Para o GSC, a experiência com o simulador é considerada positiva, visto que ele se aproxima muito da realidade, e prepara para o atendimento do paciente real, conforme evidenciado nas falas: Foi muito boa devido à interação, comunicação e acreditar que o simulador é um paciente real. (A2); Foi uma experiência muito importante para a interação com o paciente real, acredito que esta simulação nos dá a ideia de como será o contato no hospital... (A35).
As palavras "não e mais" estão relacionadas à capacidade de reflexão e autopercepção do aluno quanto ao seu conhecimento e preparo, antes do primeiro contato com o paciente no ambiente hospitalar. Não me sinto preparada ainda, sinto que preciso fixar mais o conhecimento adquirido nas aulas, treinar mais... (A19); [...] Não, eu devo mudar e corrigir muitas coisas para conseguir atender corretamente o paciente real (A28). [...] o simulador traz experiências para o estudante que talvez ele não pensasse antes, que poderia passar diante do paciente real (A53).

Ao analisar o termo simulador, palavras como "experiência, interessante, legal, ansioso e realidade" têm maior proximidade. Por ser novidade, a simulação pode ter causado ansiedade nos alunos, mas, apesar desse sentimento, a experiência foi considerada positiva:[...] achei bem interessante uma experiência nova; fiquei um pouco ansiosa... (A28); [...] a aula prática é bem produtiva, pois você consegue ouvir, ver o que está acontecendo e relacionar com a aula teórica. No simulador eu achei bem interessante, apesar de antes me sentir um pouco ansioso $e$ nervoso (A11).

O termo "paciente real" possui conectividade direta com a palavra "simulador", mas também apresenta ligação com termos como "comunicação, treinar, preparar e permitir". Para os alunos, a simulação possibilita uma experiência próxima ao que será encontrado no primeiro contato com o paciente real, além de ajudar no treinamento, facilitar a comunicação e a correção de erros: [...] essa experiência me possibilitou ter mais facilidade 


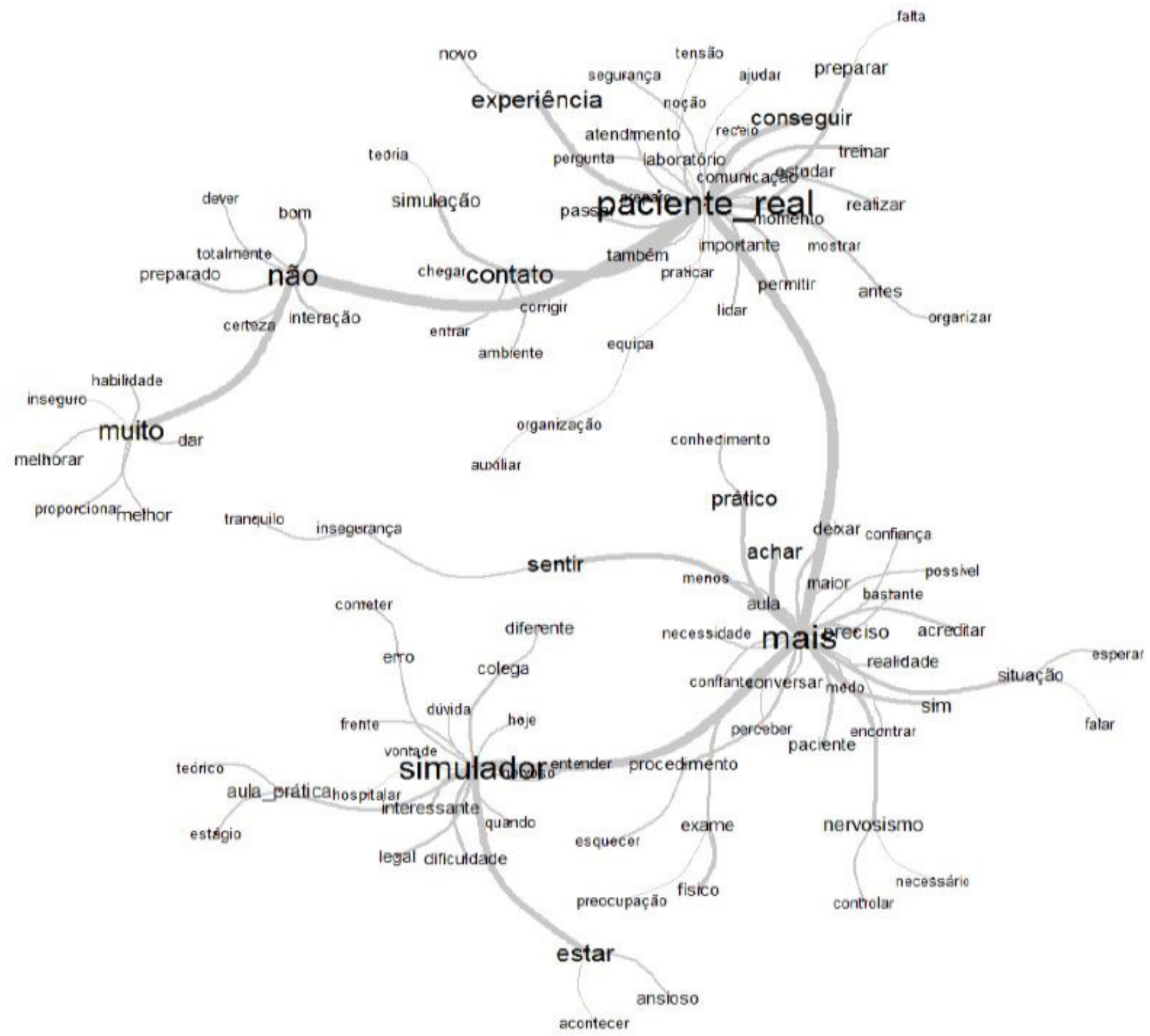

Figura 2. Árvore de similitude do Grupo Simulação Clínica. Curitiba, PR, Brasil, 2017.

Fonte: os autores (2017).

frente ao primeiro contato com o paciente real, poder ver que algumas das minhas falhas, que foram identificadas durante a aula com o simulador, foram meus pontos fortes hoje (primeira experiência clínica hospitalar) (A21).

O item lexical "não" se deve à pergunta sobre se sentir preparado para o atendimento ao paciente real. No GSC, o simulador permitiu compreender as dificuldades e a necessidade de aprofundamento nos conteúdos teóricos e aulas de laboratório antes da primeira experiência clínica, conforme evidenciado no relato: choque de realidade, a experiência foi refletida ao simulador; o nervosismo e o medo de não saber o que fazer me deixou em pânico. Não, é necessário um preparo maior tanto teórico como prático, é necessária mais aula prática (A36).

O item lexical "mais", apresentou conexão direta com a palavra "simulador", e possui em seu entorno diversas palavras, como "confiança, preciso, preparado, bastante, deixar e nervosismo". $\mathrm{Na}$ análise do corpus, observamos que os alunos sentem que o simulador prepara e dá mais confiança no fazer; contudo, também causa nervosismo, já que, como dito anteriormente, é uma experiência nova e possui características altamente reais: [...] acredito que, se fosse implementada a simulação na aula prática, os alunos chegariam no primeiro dia de estágio mais 
confiantes (A5); Acho que preciso controlar mais meu nervosismo, minha técnica; a experiência com o simulador me ofereceu uma certa experiência de como seria hoje (primeira experiência clínica hospitalar), pois no dia do simulador o enfermeiro e o professor apontaram nossos erros e hoje já os corrigi (A28).

A análise de similitude do GPC (Figura 3) apresentou três itens lexicais principais: "mais, aula prática e paciente real". Destes, o termo "mais" evidenciou o maior número de conexões. Diversas palavras possuem conexão bem próxima ao vocábulo "mais", entre elas "praticar, professor, aprender, sentir, segurança, colega, fácil".

O item lexical "mais" aparece como o eixo principal com maior número de conectividades. As mais fortes e que se encontram na periferia são os termos "não, sentir, muito, aula prática e paciente real", e cada um deles possui ligações fortes com outros termos. Nas ligações mais fortes com o vocábulo "mais", estão as palavras "seguro, conhecimento, professor, praticar, experiência, antes, melhor, treinar, realizar", entre outras.

Próximos à palavra "não", estão os termos "prático, colega, ainda, exame", entre outros. Já o item lexical "sentir" possui conexão direta com os termos "preciso, confiante, bom, conhecer, tornar e começar". "Muito" está diretamente conectado a "produtivo, preparado, importante, conseguir e aula". "Aula prática" tem ligações com "bastante, laboratório e cuidar". Por fim, o termo "paciente real" possui forte conectividade com "sim, contato, facilitar e diferente".

Para o GPC, o termo "mais" traz o significado de quantidade e intensidade, apontando que a aula prática convencional em

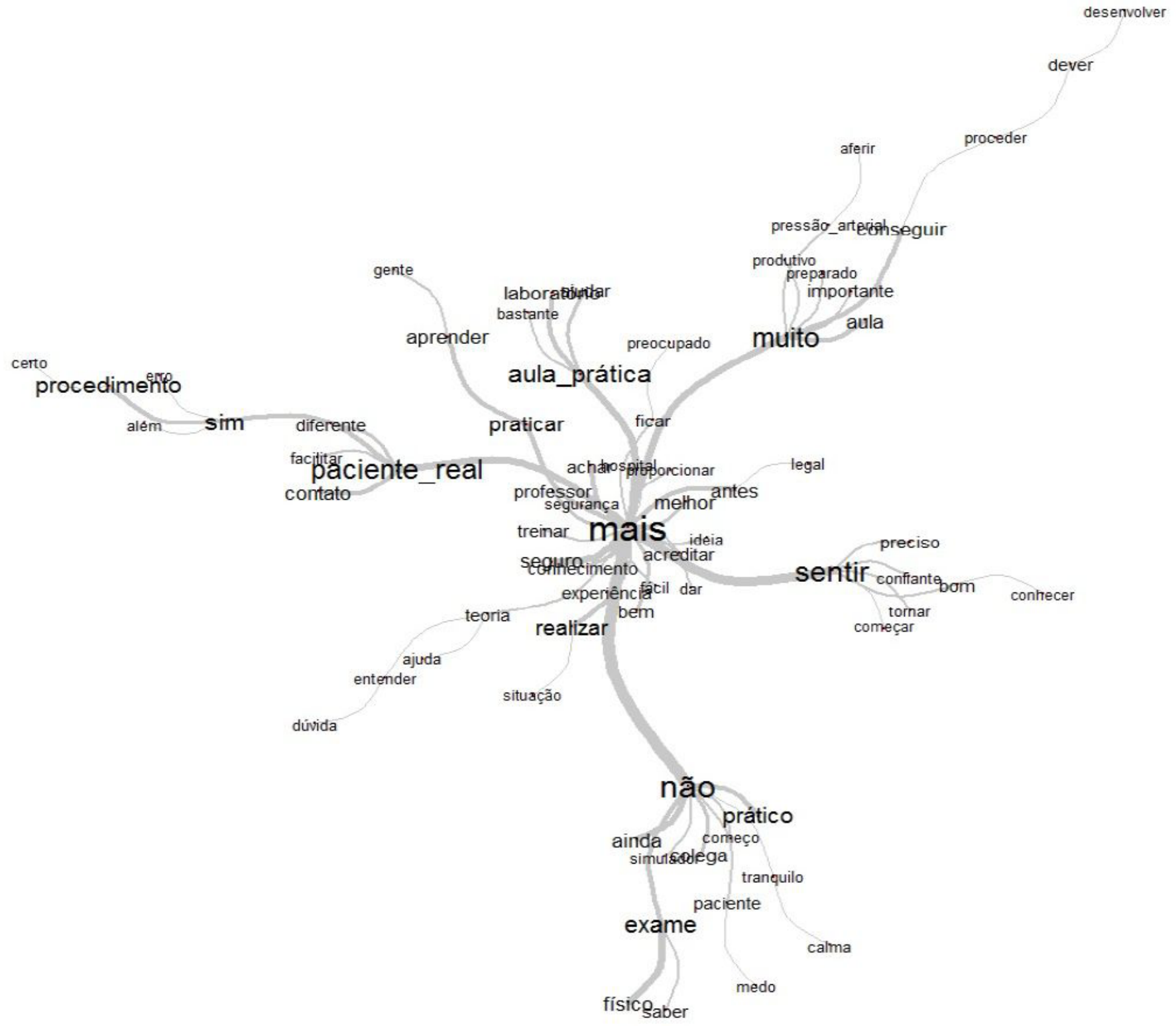

Figura 3. Árvore de similitude do Grupo Prática Convencional. Curitiba, PR, Brasil, 2017. Fonte: os autores (2017). 
laboratório ajuda na aquisição de habilidades necessárias para o atendimento seguro ao paciente. $O$ aluno se sente confortável, pois conta com a presença direta do professor, que o auxilia e demonstra como fazer os procedimentos. Ademais, eles treinam uns nos outros, e a relação de amizade favorece um ambiente tranquilo.

A quantidade de aula prática em laboratório interfere diretamente na confiança dos alunos: $A$ aula prática proporcionou uma melhor visão para o exame físico, mas ainda não me sinto segura para fazer os procedimentos, acredito que com mais aula prática essa realidade mude... (A8); [...] treinar com o colega é mais fácil e também têm os professores que estão ao seu lado (A37). O item lexical "aula prática" faz conexões com as palavras "importante, ajudar, conseguir", entre outras: $A$ aula prática foi importante porque a gente pode aprender e praticar um no outro antes de ter o contato com o paciente real... (A16).

O item lexical "paciente real" apresentou conectividade com as palavras "diferente, saber e contato". Ao analisar essas conexões, observamos que os alunos reconhecem que existe diferença entre aprender examinando colegas e examinar o paciente real: Eu não fui para a aula com o simulador e sim com um colega. E foi muito diferente, pois o meu colega eu vejo sempre e tenho um pouco de intimidade; já com o paciente real não (A6); [...] fazer exames no laboratório é bem diferente do que fazer com paciente real com dor (A37).

\section{DISCUSSÃO}

A inserção do estudante de enfermagem no campo de prática clínica é acompanhada de dificuldades na associação entre a teoria e a prática, conforme relatado por eles, a exemplo das atividades práticas pouco vivenciadas e pouco efetivas. Além disso, os estudantes demonstram ansiedade e a sensação de não terem aprendido o suficiente para vivenciar a prática clínica. ${ }^{17}$ Esse fato foi evidenciado nesta pesquisa pelos dois grupos; no entanto, observou-se que os estudantes que participaram da simulação puderam refletir sobre seus reais conhecimentos e habilidades, percebendo os pontos a serem melhorados e compreendendo a importância de se revisar os conteúdos e desenvolver competências antes da primeira experiência clínica.

Dentre os fatores que contribuem para a segurança ou insegurança do graduando de enfermagem durante as primeiras experiências clínicas, a quantidade de aulas práticas aparece como um dos principais indicadores que impactam a autoconfiança do estudante. ${ }^{18}$ Conforme apontado nesta pesquisa, eles perceberam - afora a necessidade de mais aulas práticas - a diferença que existe quando o treinamento é realizado entre colegas e com simuladores. Estes últimos trazem realismo e se aproximam muito mais da prática clínica, além de favorecer o desenvolvimento de habilidades de comunicação e a correção de erros antes do contato com o paciente.

Estudo transversal descritivo, com uso da simulação clínica após a aula teórica, constatou que os estudantes consideraram forte a influência do ensino simulado no processo de aprendizagem. Concordaram que a simulação pode ser utilizada para aumentar a autoconfiança, e recomendaram a prática simulada para outros estudantes, ${ }^{19}$ corroborando com os achados desta pesquisa em relação ao aumento da confiança, quando utilizada tal estratégia antes da primeira experiência clínica.

Em comparação com o ensino convencional, a simulação apresenta vantagens na transição para as práticas clínicas, sendo observadas maior satisfação e autoconfiança dos estudantes. ${ }^{19,20} \mathrm{~A}$ autoconfiança é um importante elemento para a tomada de decisões rápidas do enfermeiro; mais confiante, o estudante torna-se apto a resolver problemas de forma eficaz, o que minimiza a possibilidade de erros em um paciente real. ${ }^{20-22}$

Estudo realizado na Turquia, envolvendo 227 estudantes de graduação em enfermagem, comparou o ensino convencional em pediatria com o ensino baseado em simulação, e observou que os níveis de autoeficácia percebidos pelos estudantes que participaram desse tipo de ensino foram significativamente maiores. Os autores ainda destacaram que a simulação aumentou as habilidades práticas relacionadas à avaliação clínica e aos cuidados de higiene e administração de medicamentos em pediatria por parte dos estudantes ${ }^{22}$.

A simulação clínica auxilia na formação crítico-reflexiva e no desenvolvimento de competências, mediante a integração entre teoria e prática, identificação de erros, reconhecimento de falhas e na motivação em evoluir no processo de ensinoaprendizagem. ${ }^{23,24} \mathrm{~A}$ satisfação dos estudantes com a estratégia de simulação é relatada na literatura como imprescindível para o sucesso desse método, pois está associada a maior envolvimento com o processo de aprendizagem, tornando o aluno ativo na construção de conhecimento. ${ }^{25}$

Apesar de as evidências demonstrarem que a simulação possui diversas vantagens sobre outras estratégias de ensino, observa-se que ela também provoca sentimentos de estresse e ansiedade nos estudantes, o que pode comprometer o aprendizado. ${ }^{26,27}$ Tal dado também foi encontrado nesta pesquisa. Enquanto o grupo convencional relatou a prática em laboratório com colegas como um ambiente amigável e tranquilo, os estudantes que vivenciaram a simulação relataram sentimentos de nervosismo e ansiedade durante o cenário. Esses sentimentos podem estar relacionados ao momento novo, à insegurança quanto às habilidades necessárias para execução do cenário e ao realismo deste. Sendo assim, é importante que, ao elaborar os cenários de simulação, os professores estejam atentos e busquem maneiras de minimizar tais sentimentos.

A literatura recomenda: cenários adequados ao nível de conhecimento e habilidades dos estudantes; orientações claras sobre os objetivos dos cenários; tempo suficiente oportunizado para o reconhecimento do ambiente e materiais; número reduzido de participantes em cada cenário, quando possível; privacidade garantida; e preferência de observação à distância por professores e facilitadores quando a simulação se tratar de um momento avaliativo. ${ }^{28}$

Apesar das vantagens trazidas neste estudo comparativo, é imprescindível ressaltar que a simulação clínica não tem por objetivo substituir o ensino convencional, e sim complementá- 
lo. Essa estratégia se configura uma importante ferramenta de integração entre a teoria e a prática, que possibilita ao aluno uma experiência clínica interativa e segura e corrobora com as tendências de construção de ensino híbrido, utilizando múltiplos métodos de aprendizagem. ${ }^{29}$

Algumas limitações devem ser consideradas: os participantes realizaram apenas um cenário de simulação antes da primeira experiência clínica; a aula em laboratório de habilidades e o cenário de SC envolveram apenas a temática de exame físico cardiotorácico, que é parte da disciplina Fundamentos do Cuidar em Enfermagem. Contudo, na primeira experiência clínica, o conteúdo aplicado corresponde à anamnese e ao exame físico completos.

\section{CONCLUSÃO}

Na percepção dos estudantes, as duas estratégias contribuíram de forma positiva para a primeira experiência clínica com o paciente real. Porém, a simulação clínica de alta fidelidade permitiu o aprendizado em ambiente controlado e sem riscos para o paciente, em um cenário muito próximo do real. Contribuiu, ainda, para a formação de uma visão crítico-reflexiva sobre suas próprias competências, o reconhecimento das suas limitações, e a compreensão da importância e necessidade de obterem conhecimento teórico consistente para fundamentar a prática.

Este estudo fortalece as evidências dos benefícios proporcionados pelo ensino baseado em simulação, e a importância das instituições educacionais fazerem uso adequado dessa estratégia pedagógica.

\section{CONTRIBUIÇÕES DOS AUTORES}

Desenho do estudo. Radamés Boostel. Carina BortolatoMajor. Jorge Vinícius Cestari Felix.

Coleta ou produção dos dados. Radamés Boostel.

Análise de dados. Radamés Boostel. Nilton Orlando da Silva. Amanda Carolina de Oliveira Bialetzki Fontoura. Jorge Vinícius Cestari Felix.

Interpretação dos resultados. Radamés Boostel. Nilton Orlando da Silva. Jéssica de Oliveira Veloso Vilarinho. Amanda Carolina de Oliveira Bialetzki Fontoura. Jorge Vinícius Cestari Felix.

Redação e revisão crítica do manuscrito. Radamés Boostel. Carina Bortolato-Major. Nilton Orlando da Silva. Jéssica de Oliveira Veloso Vilarinho. Amanda Carolina de Oliveira Bialetzki Fontoura. Jorge Vinícius Cestari Felix.

Aprovação da versão final do artigo. Radamés Boostel. Carina Bortolato-Major. Nilton Orlando da Silva. Jéssica de Oliveira Veloso Vilarinho. Amanda Carolina de Oliveira Bialetzki Fontoura. Jorge Vinícius Cestari Felix.

Responsabilidade por todos os aspectos do conteúdo e a integridade do artigo publicado. Radamés Boostel. Carina Bortolato-Major. Nilton Orlando da Silva. Jéssica de Oliveira Veloso Vilarinho. Amanda Carolina de Oliveira Bialetzki Fontoura. Jorge Vinícius Cestari Felix.

\section{EDITOR ASSOCIADO}

\author{
Candida Primo Cançali
}

\section{REFERÊNCIAS}

1. Jamshidi N, Molazem Z, Sharif F, Torabizadeh C, Kalyani MN. The challenges of nursing students in the clinical learning environment: a qualitative study. ScientificWorldJournal. 2016;2016:1846178. http:// dx.doi.org/10.1155/2016/1846178.

2. Cabete D, Alves P, Baixinho C, Rafael H, Viegas L, Oliveira CS. A primeira experiência clínica do estudante de enfermagem. Pens Enfer [Internet]. 2016; [citado 2020 ago 4];20(2):3-25. Disponível em: http:// pensarenfermagem.esel.pt/files/Doc1_3_25.pdf

3. Alzayyat A, Al-Gamal E. A review of the literature regarding stress among nursing students during their clinical education. Int Nurs Rev. 2014;61(3):406-15. http://dx.doi.org/10.1111/inr.12114.

4. Martins JCA. Aprendizagem e desenvolvimento em contexto de prática simulada. Rev Enferm Ref. 2017;IV Série(12):155-62. http://dx.doi. org/10.12707/RIV16074

5. Lee J, Oh PJ. Effects of the use of high-fidelity human simulation in nursing education: a meta-analysis. J Nurs Educ. 2015;54(9):501-7. http://dx.doi.org/10.3928/01484834-20150814-04.

6. Omer T. Nursing students' perceptions of satisfaction and self-confidence with clinical simulation experience. J Educ Pract [Internet]. 2016; [citado 2020 ago 4];7(5):131-8. Disponível em: https://www.iiste.org/Journals/ index.php/JEP/article/view/28773

7. Gaba DM. The future vision of simulation in health care. Qual Saf Health Care.2004;13(Suppl. 1):2-10. http://dx.doi.org/10.1136/qshc.2004.009878.

8. Coutinho VRD, Martins JCA. Simulation in the education of health professionals. Rev Min Enferm. 2015;19(1):11-2. http://dx.doi. org/10.5935/1415-2762.20150001.

9. Bergamasco EC, Murakami BM, Cruz DALM. Uso da Escala de Satisfação dos Estudantes e Autoconfiança com a Aprendizagem (ESEAA) e da Escala do Design da Simulação (EDS) no ensino de enfermagem: relato de experiência. Sci Med. 2018;28(3):12. http:// dx.doi.org/10.15448/1980-6108.2018.3.31036.

10. Lubbers J, Rossman C. The effects of pediatric community simulation experience on the self-confidence and satisfaction of baccalaureate nursing students: a quasi-experimental study. Nurse Educ Today. 2016;39:93-8. http://dx.doi.org/10.1016/j.nedt.2016.01.013.

11. Ferreira RPN, Guedes HM, Oliveira DWD, de Miranda JL. Realistic simulation as a method of teaching in the learning of the health field students. Rev Enferm Cent-Oeste Min. 2018;8:e2508. http://dx.doi. org/10.19175/recom.v8i0.2508.

12. Negri EC, Mazzo A, Martins JCA, Pereira GA Jr, Almeida RGS, Pedersol CE. Clinical simulation with dramatization: gains perceived by students and health professionals. Rev Latinoam Enferm. 2017;25:e2916. http:// dx.doi.org/10.1590/1518-8345.1807.2916.

13. Buxton M, Phillippi JC, Collins MR. Simulation: a new approach to teaching ethics. J Midwifery Womens Health. 2015;60(1):70-4. http:// dx.doi.org/10.1111/jmwh.12185.

14. Bortolato-Major C, Arhur JP, Mattei AT, Mantovani MF, Felix JVC, Booste R. Contributions of the simulation for undergraduate nursing students. J Nurs UFPE. 2018;12(6):1751-62. http://dx.doi.org/10.5205/19818963-v12i6a230633p1751-1762-2018.

15. Groom JA, Henderson D, Sittner BJ. NLN/Jeffries simulation framework state of the science project: simulation design characteristics. Clin Simul Nurs. 2014;10(7):337-44. http://dx.doi.org/10.1016/j.ecns.2013.02.004

16. Camargo BV, Justo AM. IRAMUTEQ: um software gratuito para análise de dados textuais. Temas Psicol. 2013;21(2):513-8. http://dx.doi. org/10.9788/TP2013.2-16.

17. Lima JA, Carrilho AB, Santos LD, Silva de Lima UT. Expectativas do estágio hospitalar para estudantes de enfermagem. Rev Bras Ciências da Saúde.2016;14(48):5-10. http://dx.doi.org/10.13037/ras.vol14n48.3372.

18. Santos KD, De Assis MA. Fatores que contribuem para a segurança $e$ insegurança do graduando de enfermagem durante o estágio. Enferm Bras [Internet]. 2017; [citado 2020 ago 4];16(1):4-10. Disponível em 
https://portalatlanticaeditora.com.br/index.php/enfermagembrasil/ article/view/898/1851

19. Fernandes AKC, Ribeiro LM, Brasil GC, Magro MCS, Hermann PRS, Leon CGRMP, Viduedo AFS, Funghetto SS. Simulation as a strategy for learning in pediatrics. Rev Min Enferm. 2016;20:e976. http://dx.doi. org/10.5935/1415-2762.20160046.

20. Rosa MEC, Pereira-Ávila FMV, Góes FGB, Pereira-Caldeira NMV, Sousa LRM, Goulart MCL. Positive and negative aspects of clinical simulation in nursing teaching. Esc Anna Nery. 2020;24(3):e20190353. http://dx.doi.org/10.1590/2177-9465-ean-2019-0353.

21. Presado MHCV, Colaço S, Rafael H, Baixinho CL, Félix I, Saraiva C, Rebelo I. Learning with High Fidelity Simulation. Cien Saude Colet. 2018;23(1):51-9. http://dx.doi.org/10.1590/1413-81232018231.23072017. PMid:29267811.

22. Arslan FT, Türkmen AS, Çelen R, Özkan S, Altıparmak D, Şahin A Comparing traditional and simulation-based experiences in pediatrics with undergraduate nursing students in Turkey. Clin Simul Nurs. 2018;16:62-9. http://dx.doi.org/10.1016/j.ecns.2017.10.012.

23. Kim J, Park JH, Shin S. Effectiveness of simulation-based nursing education depending on fidelity: a meta analysis. BMC Med Educ. 2016;16(1):152. http://dx.doi.org/10.1186/s12909-016-0672-7.
24. INACSL Standards Committee. INACSL Standards of Best Practice: SimulationSM Simulation-Enhanced Interprofessional Education (SimIPE). Clin Simul Nurs. 2016;12(Suppl.):S34-8. http://dx.doi.org/10.1016/j. ecns.2016.09.011.

25. Teixeira CRS, Pereira MCA, Kusumota L, Gaioso VP, Mello CL, Carvalho EC. Evaluation of nursing students about learning with clinical simulation. Rev Bras Enferm. 2015;68(2):284-91. http://dx.doi.org/10.1590/00347167.2015680218i.

26. Baptista RCN, Martins JCA, Pereira MFCR, Mazzo A. Students' satisfaction with simulated clinical experiences: validation of an assessment scale. Rev Lat Am Enfermagem. 2014;22(5):709-15. http:// dx.doi.org/10.1590/0104-1169.3295.2471. PMid:25493664.

27. Araújo PRS, Duarte TTP, Magro MCS. Effect of simulation for significant learning. Rev Enferm UFPE on line. 2018;12(12):3416-25. https://doi. org/10.5205/1981-8963-v12i12a237671p3416-3425-2018.

28. Nielsen B, Harder N. Causes of student anxiety during simulation: what the literature says. Clin Simul Nurs. 2013;9(11):2507-12. http://dx.doi. org/10.1016/j.ecns.2013.03.003.

29. Almeida DR, Nodari $\mathrm{CH}$, Guimarães $\mathrm{CM}$, Coutinho AOR, Bez MR. A simulação como estratégia de ensino-aprendizagem em enfermagem: uma revisão integrativa. Rev Educ Saúde. 2018;6(2):98-105. http:// dx.doi.org/10.29237/2358-9868.2018v6i2.p98-105. 